\title{
Fraccionamiento y caracterización electroforética de las proteínas de la semilla de kañihua (Chenopodium pallidicaule Aellen)
}

\section{Fractionation and electrophoretic characterization of (Chenopodium pallidicaule Aellen) kanihua seed proteins}

\begin{abstract}
Kanihua (Chenopodium pallidicaule Aellen) is a Chenopodiacea of the andean region, that contains between 15 and $19 \%$ protein, with essential amino acids. The objective of the study was to fractionate and electrophoretically characterize the proteins of kanihua seed varieties Cupi-Sayhua and Ramis. In the whole meal, the proximal analysis and fractionation were performed, and the flour was fractionated by five techniques according to Osborne solubility to obtain albumins, globulins, prolamins and glutelins. The methodology, solvents and extraction time were optimized; and the electrophoretic profiles of the fractions were identified. The highest protein content $(p \leq 0.05)$ was of kanihua flour and its protein fractions, compared to kiwicha and wheat. The highest percent yield ( $p \leq 0.05)$ during $1 \mathrm{~h}$ of sequential extraction of the protein fractions, was obtained with the Rodriguez y et.al., technique for albumins and glutelins, and with the technique described by Barba de la Rosa y et.al., for globulins and prolamins. The following results were found in Ramis and Cupi-Sayhua kanihua: albumins: $15.4 \pm 0.3$ and $15.8 \pm 0.3 \%$, globulins $75: 24.1 \pm 0.5$ and $26.3 \pm 1.0 \%$, globulins 11 S: $25.7 \pm 1.0$ and $26.7 \pm 1.0 \%$, prolamins: $9.6 \pm 0.1$ and $9.9 \pm 0.5 \%$ and g/utelins: $22.9 \pm 0.1$ and $21.5 \pm 1.4 \%$, respectively. The electrophoretic profile showed patterns similar in number of bands and differences in concentration in both varieties.
\end{abstract}

Key words: Fractionation; electrophoresis; proteins; Chenopodium pallidicaule Aellen.

\section{INTRODUCCIÓN}

La kañiwa (Chenopodium pallidicaule Aellen) se cultiva en los andes entre Perú y Bolivia, y forma parte de la dieta del poblador andino desde épocas incaicas, conteniendo mayor cantidad de proteínas que el trigo, el centeno, la cebada, el arroz y el maíz'. Es un vegetal C4 con una modalidad fotosintética de alta eficiencia en el aprovechamiento de la radiación solar y de la fijación del dióxido de carbono presente en la atmósfera, lo cual le confiere una gran adaptabilidad a distintas condiciones ambientales, como tolerancia a sequías, fuertes vientos, heladas, crecimiento rápido entre 3.000 y $4.200 \mathrm{~m}$ de altitud y en suelos arcillosos-salinos ${ }^{2-6}$.

\begin{abstract}
Gladys Moscoso-Mujica', Amparo Zavaleta', Ángel Mujica²,
\end{abstract} Marco Santos ${ }^{1}$, Robert Calixto'.

1. Laboratorio de Biología Molecular -
Facultad de Farmacia y Bioquímica - Universidad Nacional Mayor de
San Marcos, Lima 1 - Perú.
2. Escuela de Postgrado, Universidad Nacional del Altiplano, Puno - Perú.

Dirigir la correspondencia a: Gladys Moscoso-Mujica. Laboratorio de Biología Molecular - Facultad de Farmacia y Bioquímica - Universidad Nacional Mayor de San Marcos. Jr. Puno 1002. Lima 1, Perú. Tel: +513281811 . E-mail: gladysmoscoso03@gmail.com

Este trabajo fue recibido el 10 de septiembre de 2016. Aceptado con modificaciones el 21 de marzo de 2017 y aceptado para su publicación el 5 de mayo de 2017

La kañihua es una dicotiledónea que pertenece al género Chenopodium, contiene proteínas (entre 15 y 19\%) y aminoácidos esenciales como lisina (5,0 a 6,3\%), metionina $\left(1,4\right.$ a 3,0\%), treonina $(4,4$ a $4,7 \%)$ y triptófano $(0,7 \text { a } 0,9 \%)^{7,8}$. Es una buena fuente de aminoácidos esenciales, aproximándose a los valores recomendados por la $\mathrm{FAO} / \mathrm{WHO} / \mathrm{UNU}^{9}$. Además, posee vitaminas, minerales, polisacáridos y azúcares libres en pequeñas cantidades, alto contenido de fibra insoluble, lípidos con omega 3, 6 y 9; y saponinas que no presentan el sabor amargo a diferencia de otros granos andinos como la quinua y el tarwi ${ }^{10,11}$.

En diversas investigaciones se han encontrado compuestos 
y moléculas como proteínas de cereales, leguminosas, granos andinos, verduras, frutas y otros alimentos naturales, con propiedades de retrasar la progresión de enfermedades, inhibir los mecanismos fisiopatológicos o suprimir las actividades de patógenos, entre otros ${ }^{12,13}$. Por lo tanto, el rol que desempeñan las proteínas como componentes fisiológicamente activos se está reconociendo cada vez más en los últimos años, principalmente porque proporcionan una fuente de péptidos biológicamente activos ${ }^{14}$.

La kañihua se caracteriza por su contenido proteínico de elevada calidad y cantidad nutricional. Sin embargo, el valor nutricional de este grano no ha sido estudiado exhaustivamente ${ }^{7,8,13}$. Además, en la búsqueda de proteínas como fuentes de péptidos bioactivos, los estudios se han centrado en las proteínas del trigo, soya, kiwicha (Amaranthus), quinua, pero pocos en kañihua ${ }^{12-21}$, utilizando principalmente concentrados proteicos ${ }^{2,13,15}$ : y ninguna investigación emplean fracciones proteicas.

La kañihua presenta diversas proteínas que se pueden separar con base en su solubilidad según el criterio de Osborne, por ejemplo, las albuminas son solubles en agua, las globulinas en soluciones salinas diluidas, las prolaminas en soluciones alcohólicas, y las glutelinas en soluciones ácidas o alcalinas ${ }^{22}$. Las diferentes proteínas presentan elevado contenido proteínico y son fuentes potenciales de péptidos bioactivos con propiedades funcionales y nutracéuticas ${ }^{12-15}$. El objetivo de esta investigación fue obtener el fraccionamiento y caracterización electroforética de las proteínas de la semilla de kañihua de las variedades Ramis y Cupi-Sayhua, a través de la evaluación de cinco técnicas de fraccionamiento proteico, según la solubilidad de Osborne, considerando solventes, metodologías y tiempo de extracción, para obtener fracciones proteicas con mayores contenidos proteínicos y rendimientos porcentuales, que incentiven a investigaciones posteriores a desarrollar nutracéuticos ricos en péptidos bioactivos.

\section{MATERIAL Y MÉTODOS}

Preparación de harina

Los granos de Chenopodium pallidicaule Aellen variedad Ramis y Cupi-Sayhua se obtuvieron de la Universidad Nacional del Altiplano, Puno, Perú. Los granos se limpiaron y molieron en el molino estacionario Thomas Willey (Arthur H. Thomas Company, USA), luego se tamizaron para obtener partículas de 0,250 $\mathrm{mm}$. La harina integral fue deslipidizada con n-hexano en proporción 1:10 (p/v) $)^{18}$ con agitación a $4{ }^{\circ} \mathrm{C}$ durante $24 \mathrm{~h}^{23}$, luego se secó a temperatura ambiente por 24 h, se tamizó y conservó en frascos ámbar a $4{ }^{\circ} \mathrm{C}$.

\section{Composición proximal}

La composición proximal de la harina integral y deslipidizada fue determinada de acuerdo a los procedimientos oficiales, descritos en $\mathrm{AOAC}^{24}$. La humedad (método 934.01) se determinó con base a la diferencia de peso al ser secada en horno a $110^{\circ} \mathrm{C}$ durante $2 \mathrm{~h}$. El nitrógeno (978.04) según el sistema de Kjeldahl. La proteína fue calculada del nitrógeno total usando el factor de 5,87 reportado para kiwicha ${ }^{18,20}$.
Las cenizas (930.05) se calcularon por peso de la muestra después de calentar a $550{ }^{\circ} \mathrm{C}$ durante $2 \mathrm{~h}$. La grasa (930.09) fue obtenida por extracción con éter de petróleo durante 4 h en el sistema Soxhlet. La fibra (934.10) se determinó por digestión con soluciones de ácido sulfúrico e hidróxido de sodio y calcinación. Los carbohidratos y el balance energético se determinaron por cálculo ${ }^{24,25}$. Estos análisis fueron realizados en el Centro de Control Analítico-UNMSM, Lima, Perú.

\section{Obtención de fracciones proteicas}

Se evaluó cinco técnicas de fraccionamiento proteínico según la solubilidad de Osborne reportados para el grano de kiwicha, difiriendo cada una en la metodología, solventes y tiempo de extracción. Todas las extracciones fueron secuenciales. Se empezó por las albuminas, seguida de las globulinas, luego las prolaminas y finalmente las glutelinas. Sólo en la fracción de las albuminas se pesó la harina y se mezcló con el solvente según la técnica, posteriormente se homogenizó con agitación constante durante el tiempo de mayor rendimiento, se prosiguió con la centrifugación a 9.000 x g por 20 min a $4{ }^{\circ} \mathrm{C}$ en una centrifuga refrigerada Eppendorf 5810R (EPPENDORF, USA) y los sobrenadantes que contenían la fracción proteica se liofilizaron en un equipo Labconco 2,5 (LABCONCO, Alemania) para los análisis subsecuentes, y los precipitados sirvieron para obtener la siguiente fracción de manera secuencial considerando las mismas condiciones de tiempo de extracción, centrifugación y liofilización. Las técnicas utilizadas fueron: 1) Maldonado-Cervantes y et.al ${ }^{26}$; 2) Barba de la Rosa y et.al. ${ }^{27}$; 3) Rodríguez y et.al ${ }^{28}$; 4) SorianoSantos y et.al ${ }^{20}$; y 5) Barba de la Rosa y et.al ${ }^{18}$.

1. Albuminas. La harina fue resuspendida en agua bi-destilada en proporción 1:10 (p/v) para las técnicas de Maldonado-Cervantes y et.al ${ }^{26}$, Barba de la Rosa y et.al ${ }^{27}$, Rodríguez y et.al ${ }^{28}$ y Barba de la Rosa y et.al ${ }^{18}$, y para la técnica de Soriano-Santos y et.al ${ }^{20}$, el solvente fue $\mathrm{NaCl}$ al $10 \%$ en proporción 1:20 (p/v). Una vez obtenido el sobrenadante conteniendo las albuminas más nitrógeno no-proteico (albuminas + NNP), para el caso de Rodríguez y et.al ${ }^{28}$, se ajustó el $\mathrm{pH}$ a 3 con $\mathrm{HCl} 2 \mathrm{~N}$ para precipitar las albuminas. De acuerdo con Soriano-Santos y et.al ${ }^{20}$, las proteínas del sobrenadante se precipitaron con $\left(\mathrm{NH}_{4}\right)_{2} \mathrm{SO}_{4}$ a los porcentajes de saturación de 50, 70 y $100 \%$, y luego fueron dializadas por $24 \mathrm{~h}$ para obtener las albuminas.

2. Globulinas. El precipitado obtenido en el paso anterior fue resuspendido en diferentes solventes en proporción de 1:10 (p/v). Según la técnica de Maldonado-Cervantes y et.al ${ }^{26}$ se obtuvo dos tipos, las globulinas $7 \mathrm{~S}$ (con el tampón $\left.\mathrm{K}_{2} \mathrm{HPO}_{4} 10 \mathrm{mM}(\mathrm{pH} 7,5), \mathrm{NaCl} 0,1 \mathrm{M}, \mathrm{EDTA} 1 \mathrm{mM}\right)$, y globulinas $11 \mathrm{~S}$ (con el tampón $\mathrm{K}_{2} \mathrm{HPO}_{4} 10 \mathrm{mM}(\mathrm{pH} 7,5), \mathrm{NaCl}$ 0,8 M, EDTA $1 \mathrm{mM})$. De acuerdo a Barba de la Rosa y et.al ${ }^{27}$ se empleó, a) $\mathrm{Na}_{2} \mathrm{HPO}_{4} 0,1 \mathrm{M}\left(\mathrm{pH}\right.$ 7); b) $\mathrm{Na}_{2} \mathrm{HPO}_{4} 0,1 \mathrm{M}(\mathrm{pH}$ 7) $\left(\mathrm{NH}_{4}\right)_{2} \mathrm{SO}_{4} \%$ (pH 7); c) $\mathrm{NaCl} 0,8 \mathrm{M} ;$ d) $\mathrm{Na}_{2} \mathrm{HPO}_{4} 0,1$ $\mathrm{M}(\mathrm{pH} 7),\left(\mathrm{NH}_{4}\right)_{2} \mathrm{SO}_{4} 5 \%$ y NaCl 0,8 M. Para el caso de la técnica de Rodríguez y et.al ${ }^{28}$ se utilizó $\mathrm{Na}_{2} \mathrm{HPO}_{4} 0,1 \mathrm{M}(\mathrm{pH}$ 7,5 ) ajustando el $\mathrm{pH}$ a 3 con $\mathrm{HCl} 2 \mathrm{~N}$. Según a la técnica de Barba de la Rosa y et.al ${ }^{18}$ se obtuvo dos tipos, las globulinas 
$7 \mathrm{~S}$ con $\mathrm{Na}_{2} \mathrm{HPO}_{4} 10 \mathrm{mM}(\mathrm{pH}$ 7,5), EDTA $1 \mathrm{mM}, \mathrm{NaCl} 0,1$ $\mathrm{M}$, y globulinas $11 \mathrm{~S}$ con $\mathrm{Na}_{2} \mathrm{HPO}_{4} 10 \mathrm{mM}(\mathrm{pH}$ 7,5), EDTA 1 $\mathrm{mM}$ y $\mathrm{NaCl} 0,8 \mathrm{M}$.

3. Prolaminas. De acuerdo a las técnicas de Barba de la Rosa y et.al ${ }^{27}$, Rodríguez y et.a ${ }^{28}$ y Barba de la Rosa y et.a $\left.\right|^{18}$ se obtuvo el precipitado de las globulinas el que fue resuspendido en 2-propanol 70\% en la proporción de 1:10 $(\mathrm{p} / \mathrm{v})$.

4. Glutelinas. Se resuspendió el precipitado de las globulinas $11 \mathrm{~S}$ según la técnica de Maldonado-Cervantes y et.al ${ }^{26}$, y el precipitado de las prolaminas en las demás técnicas, en la proporción de 1:10 (p/v). De acuerdo a Maldonado-Cervantes y et.a ${ }^{26}$ y Rodríguez y et.a ${ }^{28}$ se usó $\mathrm{NaOH} 0,1 \mathrm{M}$. Según la técnica de Barba de la Rosa y et.al ${ }^{27}$ se empleó, a) $\mathrm{NaOH} 0,1 \mathrm{M}$, b) EDTA $1 \mathrm{mM}$, c) $\mathrm{Na}_{2} \mathrm{HPO}_{4} 0,1$ $\mathrm{M}(\mathrm{pH} 7), \mathrm{d}) \mathrm{HCl}$ 0,1 M. Para el caso de la técnica de Barba de la Rosa y et.al ${ }^{18}$ se usó $\mathrm{Na}_{2} \mathrm{HPO}_{4} 10 \mathrm{mM}$ (pH 7,5), EDTA $1 \mathrm{mM}$ y $\mathrm{NaOH}$ 0,1 mM.

\section{Tiempo de extracción}

Con las técnicas que se obtengan mayores rendimientos porcentuales de extracción de las fracciones proteicas, se evaluó el mejor tiempo de extracción secuencial de fracciones proteicas de kañihua Ramis y Cupi-Sayhua a $4{ }^{\circ} \mathrm{C}$ y agitación constante. Se empleó $1 \mathrm{~h}$ para extraer albuminas, $1 \mathrm{~h}$ para globulinas 7S, $1 \mathrm{~h}$ para globulinas $11 \mathrm{~S}, 1 \mathrm{~h}$ para prolaminas, y $1 \mathrm{~h}$ para glutelinas. De la misma manera se prosiguió con 2,4 y 8 h. Elegido el tiempo óptimo de la extracción, este se empleó para la obtención de todas las fracciones proteicas secuenciales.

Determinación del contenido proteico

El contenido de proteínas solubles en cada fracción, se determinó por el método descrito por Bradford ${ }^{29}$, y se utilizó como patrón albúmina sérica de bovino.

\section{Electroforesis}

Las proteínas de las diversas fracciones se diferenciaron por geles de poliacrilamida al $10 \%$ en presencia de dodecilsulfato de sodio (SDS-PAGE) de acuerdo al método de Laemmli ${ }^{30}$, usando el equipo Mini Omni PAGE (Cleaver, USA) a 90 y $100 \mathrm{~V}$ para los geles de concentración y separación, respectivamente. Luego, se tiñeron con azul brillante de Coomassie R-250. Los pesos moleculares se determinaron usando los marcadores de peso molecular (MP) Broad range (6,5 a $200 \mathrm{kDa})$ y Dual color (10 a $250 \mathrm{kDa}$ ) (BIO-RAD).

\section{Rendimiento en la obtención de las fracciones proteínicas}

Para el cálculo del rendimiento de extracción de las fracciones proteínicas de kañihua Ramis y Cupi-Sayhua, se tomó como referencia el contenido de la proteína en la harina determinada en el análisis proximal (proteína cruda), de acuerdo a la formula reportada por Gomez ${ }^{31}$.
Análisis estadístico

El análisis fue realizado utilizando el programa estadístico STATA versión 11,0 (Stata Corp LP, College Station, Texas). Para el análisis diferencial de medias se utilizó chi cuadrado y ANOVA con pruebas post-hoc. Se consideró un intervalo de confianza al $95 \%$ y a todo valor de $p \leq 0,05$ como estadísticamente significativo. Todos los análisis fueron realizados por triplicado, se expresaron como la media $(\mu)$ \pm error estándar de la muestra (SEM).

\section{RESULTADOS}

Análisis proximal

Los contenidos proteínicos de la harina integral de

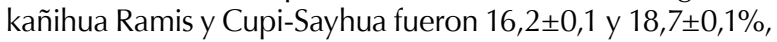
respectivamente, valores mayores en comparación al grano andino kiwicha y cereal trigo. Se observó incremento del contenido proteínico en la harina deslipidizada en aproximadamente un $10 \%$. Además, destacó la proporción de grasa de kañihua y kiwicha en comparación al trigo (Tabla 1).

\section{Fracciones proteicas}

El contenido proteínico de la harina integral de kañihua fue mayor en la variedad Cupi-Sayhua, siendo la fracción con mayor contenido proteínico las albuminas + NNP, seguida de las globulinas, glutelinas, y finalmente las prolaminas (Tabla 2). Las fracciones albuminas + NNP y globulinas de kañihua, presentaron, mayor contenido proteínico $(p \leq 0,05)$ en comparación a kiwicha y trigo.

Luego, se prosiguió a determinar el rendimiento porcentual de las fracciones proteicas de la harina de kañihua empleando cinco técnicas de fraccionamiento (Figura 1). Para fines de la investigación no se consideraran a las albuminas + NNP, sólo a las albuminas, por lo tanto, se observó mayor rendimiento $(p \leq 0,05)$ de extracción proteínica en las fracciones albuminas y glutelinas con la técnica de Rodríguez y et.al ${ }^{28}$, y en las fracciones globulinas y prolaminas con la técnica Barba de la Rosa y et.al ${ }^{18}$ en comparación a las fracciones obtenidas con la técnica de Maldonado-Cervantes y et.al ${ }^{26}$, Barba de la Rosa y et.al ${ }^{27}$, Soriano-Santos y et.a ${ }^{20}$. De esta manera, se consideró los solventes y metodología de estas dos técnicas como óptimos para extraer las fracciones proteínicas de kañihua en las dos variedades evaluadas.

Continuando con la evaluación de las técnicas de extracción de las fracciones proteicas de kañihua, en la evaluación del tiempo de extracción se observó que no existieron diferencias significativas en el rendimiento porcentual de las fracciones proteicas según el tiempo de extracción de 1, 2, 4 y 8 h. Por lo tanto, se consideró el menor tiempo de 1 h como el óptimo en la obtención de las fracciones proteicas (Figura 2).

Finalmente, con los solventes, metodología y tiempo óptimo para la extracción de las fracciones proteicas de kañihua (descritos líneas arriba), se obtuvo un mayor contenido proteico y rendimiento porcentual $(p \leq 0,05)$ de albuminas y glutelinas fraccionadas con la técnica de Rodríguez y et.al ${ }^{28}$, y globulinas $7 \mathrm{~S}, 11 \mathrm{~S}$ y prolaminas con la técnica de Barba de 


\section{TABLA 1}

Composición proximal de la harina integral de kañihua (Chenopodium pallidicaule Aellen) de las variedades Ramis y Cupi-Sayhua en comparación a otros granos.

\begin{tabular}{|c|c|c|c|c|c|c|c|}
\hline \multirow[b]{2}{*}{ Parámetros } & \multicolumn{2}{|c|}{ Kañihua Ramis } & \multicolumn{2}{|c|}{$\begin{array}{c}\text { Kañihua } \\
\text { Cupi-Sayhua }\end{array}$} & \multirow[t]{2}{*}{ Quinua* } & \multirow[t]{2}{*}{ Kiwicha* } & \multirow[t]{2}{*}{ Trigo* } \\
\hline & I & D & I & D & & & \\
\hline Humedad (\%) & $11,6 \pm 0,1$ & $14,1 \pm 0,1$ & $11,0 \pm 0,1$ & $13,8 \pm 0,1$ & 12,2 & 11,5 & 10,26 \\
\hline Proteínas (\%) & $16,2 \pm 0,1$ & $18,2 \pm 0,1$ & $18,7 \pm 0,1$ & $20,8 \pm 0,1$ & 14,4 & 14,5 & 13,7 \\
\hline Grasas (\%) & $6,0 \pm 0,0$ & $0,6 \pm 0,0$ & $4,5 \pm 0,0$ & $0,5 \pm 0,0$ & 5,3 & 5,7 & 2,1 \\
\hline Fibra (\%) & $5,8 \pm 0,0$ & $12,5 \pm 0,1$ & $5,5 \pm 0,0$ & $14,1 \pm 0,2$ & 3,5 & 4,4 & 2,5 \\
\hline Cenizas (\%) & $4,1 \pm 0,0$ & $7,4 \pm 0,1$ & $4,5 \pm 0,1$ & $6,8 \pm 0,1$ & 2,6 & 2,3 & 1,6 \\
\hline Carbohidratos (\%) & $56,3 \pm 0,0$ & $47,2 \pm 0,0$ & $55,7 \pm 0,0$ & $43,9 \pm 0,0$ & 63,7 & 63,3 & 72,57 \\
\hline Nitrógeno (\%) & $2,8 \pm 0,0$ & $3,1 \pm 0,0$ & $3,2 \pm 0,0$ & $3,5 \pm 0,0$ & ---- & ---- & ---- \\
\hline Energía (Kcal) & $344,3 \pm 0,0$ & $267,0 \pm 0,0$ & $338,1 \pm 0,0$ & $263,6 \pm 0,0$ & ---- & ---- & 339,0 \\
\hline ELN & $67,9 \pm 0,0$ & $61,3 \pm 0,0$ & $66,7 \pm 0,0$ & $57,9 \pm 0,0$ & ---- & ---- & ---- \\
\hline
\end{tabular}

Datos expresados en base seca. Harinas: Integral (I), Deslipidizada (D). ELN (Extracto Libre de Nitrógeno). *Fuente: Mujica y Chura7, Repo-Carrasco ${ }^{8}$, Hernández-Ledesma ${ }^{12}$.

TABLA 2

Contenido proteínico en las fracciones proteicas de la harina integral y deslipidizada de kañihua (Chenopodium pallidicaule Aellen) de las variedades Ramis y Cupi-Sayhua (g/100 g de harina seca).

\begin{tabular}{|c|c|c|c|c|c|c|c|c|}
\hline \multirow{3}{*}{ Harina } & \multicolumn{6}{|c|}{ Fracciones Proteicas } & & \\
\hline & \multicolumn{2}{|c|}{ Albuminas+NNP } & \multicolumn{2}{|c|}{ Globulinas } & \multicolumn{2}{|c|}{ Prolaminas } & \multicolumn{2}{|c|}{ Glutelinas } \\
\hline & I & D & I & D & I & D & I & D \\
\hline $\begin{array}{l}\text { Kañihua } \\
\text { Ramis }\end{array}$ & $4,4 \pm 0,1^{*}$ & $5,3 \pm 0,0$ & $2,8 \pm 0,1^{*}$ & $3,2 \pm 0,1$ & $1,0 \pm 0,1$ & $1,3 \pm 0,1$ & $2,2 \pm 0,4$ & $2,6 \pm 0,1$ \\
\hline $\begin{array}{l}\text { Kañihua } \\
\text { Cupi-Sayhua }\end{array}$ & $4,6 \pm 0,3^{*}$ & $7,3 \pm 0,0$ & $3,8 \pm 0,1^{*}$ & $5,2 \pm 0,4$ & $1,2 \pm 0,1$ & $1,7 \pm 0,1$ & $2,9 \pm 0,6$ & $3,3 \pm 0,7$ \\
\hline Kiwicha & $1,9 \pm 0,1$ & $3,5 \pm 0,0$ & $2,3 \pm 0,1$ & $2,8 \pm 0,2$ & $0,7 \pm 0,1$ & $0,9 \pm 0,0$ & $1,8 \pm 0,2$ & $2,1 \pm 0,3$ \\
\hline Trigo & $1,7 \pm 0,2$ & $2,9 \pm 0,0$ & $1,2 \pm 0,1$ & $2,0 \pm 0,2$ & $2,7 \pm 0,1$ & $4,1 \pm 0,0$ & $3,9 \pm 0,1$ & $4,5 \pm 0,3$ \\
\hline
\end{tabular}

la Rosa y et.al ${ }^{18}$ durante 1 h de extracción, en comparación a kiwicha y trigo (Tabla 3). Además, al combinar estas dos técnicas se obtuvo mayores rendimientos porcentuales de las fracciones proteicas $(p \leq 0,05)$ en comparación a las fracciones obtenidas con las técnicas individuales. También, se observó que las globulinas $11 \mathrm{~S}$ y $7 \mathrm{~S}$ presentaron mayores rendimientos porcentuales seguidas de glutelinas, albuminas y prolaminas, en ambas variedades de kañihua. Sin embargo, la variedad Cupi-Sayhua presentó mayor cantidad del contenido proteínico y rendimiento porcentual sin diferencia significativa.

Perfil electroforético de las fracciones proteicas de la harina de kañihua

El perfil electroforético de las fracciones proteicas obtenidas de la harina de kañihua Cupi-Sayhua, presentó en la fracción albuminas (línea 1, Figura 3) bandas intensas entre 5 a $95 \mathrm{kDa}$, con tres dobles bandas en 25, 36 y $46 \mathrm{kDa}$. En globulinas 7S (línea 2, Figura 3) se observaron bandas de menor intensidad entre 50 a $92 \mathrm{kDa}$, y de mayor intensidad entre 4 a $55 \mathrm{kDa}$, doble banda entre 31 a $35 \mathrm{kDa}$, y 21 a 27 kDa. En globulinas 11S (línea 4, Figura 3) las bandas de mayor intensidad se visualizaron entre 4 a $37 \mathrm{kDa}$, doble banda entre 31 a 34 kDa y 21 a 25 kDa. En prolaminas (línea 5, Figura 3) las bandas estuvieron entre 110 a $200 \mathrm{kDa}$, y un conjunto de bandas entre 6,5 a $23 \mathrm{kDa}$. Finalmente, glutelinas (línea 6, Figura 3) presentó las bandas de menor intensidad entre 60 a $230 \mathrm{kDa}$, y de mayor intensidad entre 6 a $37 \mathrm{kDa}$, doble banda entre 31 a $35 \mathrm{kDa}$ y 20 a $25 \mathrm{kDa}$. La harina sin fraccionar en ambas variedades de kañihua mostró mayor cantidad de bandas que las fracciones, con regiones de polipéptidos entre 


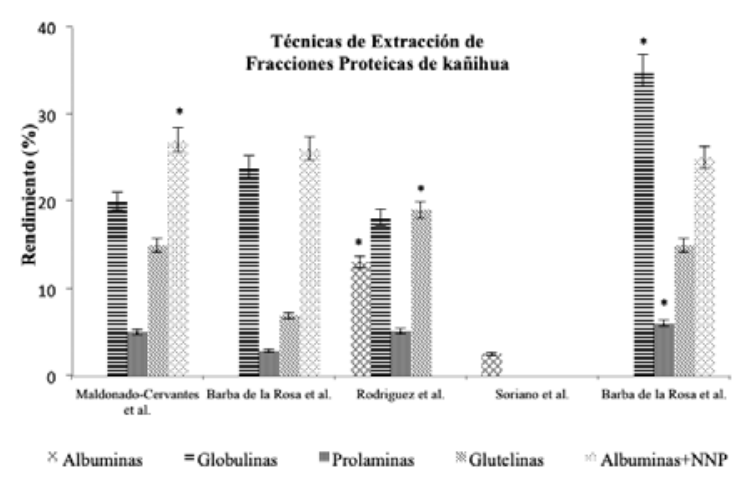

Figura 1. Rendimiento porcentual de las fracciones proteicas de la harina deslipidizada de kañihua (Chenopodium pallidicaule Aellen) de la variedad Ramis según técnicas de extracción $(\mu \pm$ SEM). Diferencia significativa $*(p \leq 0,05)$.

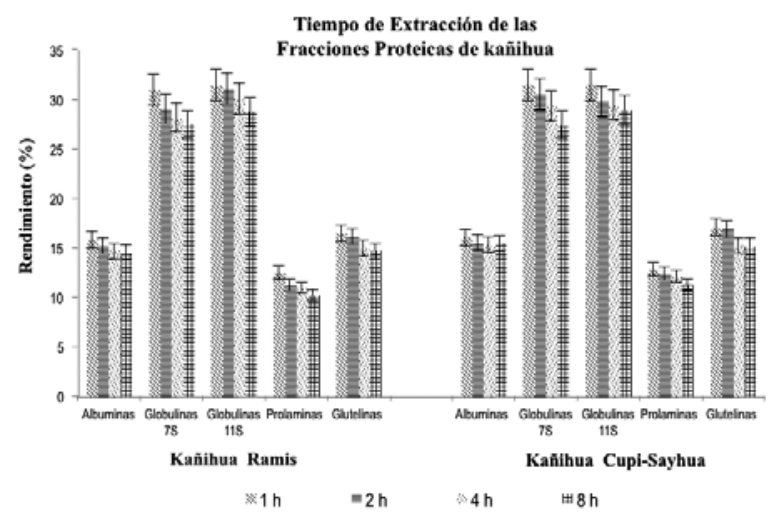

Figura 2. Rendimiento porcentual de las fracciones proteicas de la harina deslipidizada de kañihua (Chenopodium pallidicaule Aellen) de las variedades Ramis y Cupi-Sayhua a diferentes tiempos de extracción $(\mu \pm S E M)$. Fracciones extraídas con la técnica de Rodríguez y et.al ${ }^{28}$ y Barba de la Rosa y et.al ${ }^{18}$.

\section{TABLA 3}

Contenido proteínico y rendimiento porcentual en las fracciones proteicas de harina deslipidizada de kañihua (Chenopodium pallidicaule Aellen) de las variedades Ramis y Cupi-Sayhua (g/100 g harina seca)

\begin{tabular}{lcccccc} 
Fracción & \multicolumn{2}{l}{ Kañihua Ramis } & \multicolumn{2}{c}{ Kañihua Cupi-Sayhua } & Kiwicha & Trigo \\
Proteica & Proteín A & $\begin{array}{c}\text { Rendimiento } \\
\mathbf{( \% )}\end{array}$ & Proteína & $\begin{array}{c}\text { Rendimiento } \\
\mathbf{( \% )}\end{array}$ & $\begin{array}{c}\text { Rendimiento } \\
\mathbf{( \% )}\end{array}$ & $\begin{array}{c}\text { Rendimiento } \\
\mathbf{( \% )}\end{array}$ \\
Albuminas & $2,7 \pm 0,1$ & $15,4 \pm 0,3^{*}$ & $2,8 \pm 0,0$ & $15,8 \pm 0,1^{*}$ & $12,7 \pm 0,3$ & $12,0 \pm 0,3$ \\
Globulinas-7S & $4,2 \pm 0,1$ & $24,1 \pm 0,5^{*}$ & $4,6 \pm 0,2$ & $26,3 \pm 0,1^{*}$ & $19,2 \pm 1,0$ & $5,3 \pm 0,1$ \\
Globulinas-11S & $4,5 \pm 0,2$ & $25,7 \pm 1,0^{*}$ & $4,7 \pm 0,2$ & $26,7 \pm 0,1^{*}$ & $21,1 \pm 1,0$ & $4,6 \pm 0,1$ \\
Prolaminas & $1,7 \pm 0,0$ & $9,6 \pm 0,1^{*}$ & $1,7 \pm 0,1$ & $9,9 \pm 0,2^{*}$ & $6,7 \pm 0,5$ & $20,1 \pm 0,2$ \\
Glutelinas & $4,0 \pm 0,0$ & $22,9 \pm 0,1^{*}$ & $3,8 \pm 0,3$ & $21,5 \pm 0,2^{*}$ & $17,5 \pm 1,4$ & $28,2 \pm 0,2$
\end{tabular}

Fracciones proteicas extraídas con la técnica de Rodríguez y et.al ${ }^{22}$ y Barba de la Rosa y et.al ${ }^{12}$.

5 a 250 kDa, observándose mayor intensidad de las bandas en kañihua Cupi-Sayhua (Línea 8 y 9, Figura 3). Así mismo, en kañihua Ramis se observaron similar número de bandas que kañihua Cupi-Sayhua pero de menor intensidad.

\section{DISCUSIÓN}

El análisis proximal de un alimento permite determinar el valor nutritivo en composición y contenido, esta información es importante en el control de calidad del alimento, para obtener un producto final óptimo. Los granos andinos como kañihua se consideran completos por su valor nutritivo y calidad biológica, con elevada cantidad de proteína en comparación a algunos cereales. En el presente estudio la cantidad de proteína de la harina integral fue 16,2 y 18,7\% de kañihua Ramis y Cupi-Sayhua, respectivamente (Tabla
1); valores mayores a los reportados por otros autores como Giuliani y et.al ${ }^{32}$ que presentaron $16,1 \%$. También, Mujica y Chura $^{7}$ mostraron en kañihua amarilla $14,3 \%$, gris $14,0 \%$, parda $13,8 \%$ y plomiza $14,0 \%$. De manera similar, a lo descrito por Repo-Carrasco-Valencia y et.al ${ }^{36} 15,2 \%$, Callisaya y Alvarado ${ }^{2}$ en kañihua amarilla-kallutaca $14,7 \%$ y beige-taraco $13,5 \%$, y Espinosa ${ }^{13} 15,3 \%$. Las variedades de kañihua Ramis y Cupi-Sayhua presentaron un elevado contenido de proteínas en comparación a la mayoría de cereales, como el trigo que contiene entre 9,61 al 15,4\%, arroz con 7,4 a 9,9\%, y maíz con 9,2 a 13\% ${ }^{32-37}$. A la vez, el contenido de grasas de kañihua Ramis y Cupi-Sayhua fue de 6 y $4,5 \%$, respectivamente, valores similares a los reportados en kiwicha $6 \%$ y quinua $5 \%$, y mayores a cereales como trigo $2,1 \%$, maíz 4,5\% y arroz 2\% $\%^{33-35}$. Los demás parámetros del 


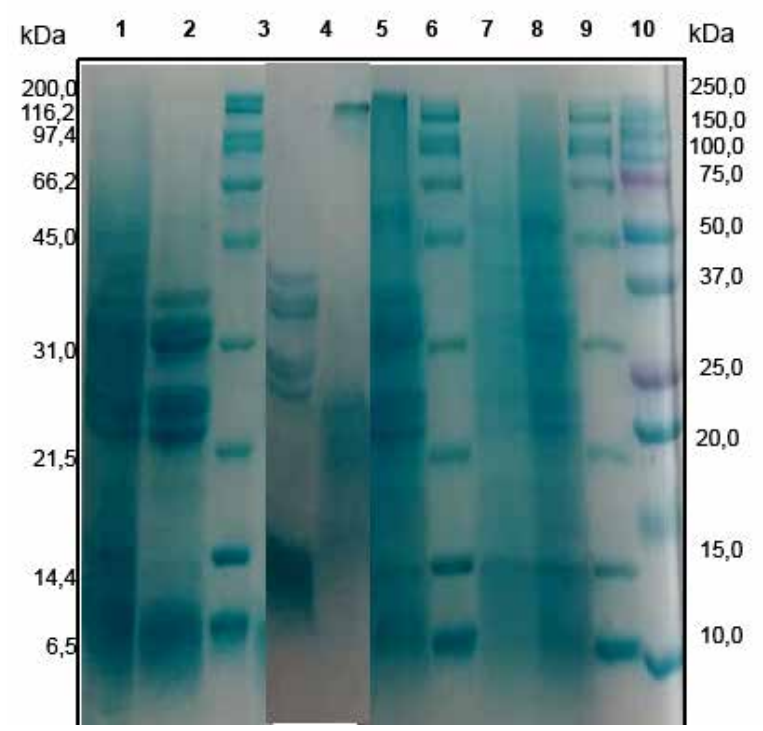

Figura 3. Electroforesis SDS-PAGE de las fracciones proteicas de kañihua (Chenopodium pallidicaule Aellen) variedad CupiSayhua. Líneas: 1, Albuminas; 2, Globulinas 7S; 3, 7 y 10, MP (Broad range); 4, Globulinas 11S; 5, Prolaminas; 6, Glutelinas; 8, Harina deslipidizada de kañihua Ramis; 9, Harina deslipidizada de kañihua Cupi-Sayhua; 11, MP (Dual color).

análisis proximal evaluado en las variedades Ramis y CupiSayhua, estuvieron dentro de los rangos descritos en otras variedades de kañihua por Mujica y Chura ${ }^{7}$ Repo-Carrasco ${ }^{8}$.

El contenido proteínico en las fracciones proteicas de la harina integral de kañihua fue mayor en comparación al grano andino kiwicha y al cereal trigo $(p \leq 0,05)$. Así mismo, se observó mayor contenido proteínico en la fracción albumina + NNP, seguida de globulinas, glutelinas y prolaminas (Tabla 2). Además, la exposición de la harina integral al solvente n-hexano por 24 h disminuyó el contenido de lípidos e incrementó la solubilidad de las proteínas en aproximadamente $10 \%$. En otras investigaciones de fraccionamiento proteico de harina integral de semilla de kiwicha, mostraron que la deslipidización incrementó el contenido de proteínas solubles entre 10 y $75 \%$, e influenció en la distribución de la solubilidad de las fracciones proteicas, siendo menor en prolamina $y$ glutelina. Utilizaron n-hexano en la proporción 1:10 (p/v) en la deslipidización por ser de fácil evaporación y bajo costo, con esta información se usó este solvente para deslipidizar la harina integral de kañihua Ramis y Cupi-Sayhua ${ }^{18,39-41}$.

El fraccionamiento proteico según la solubilidad de Osborne, permite separar fracciones y conocer la cantidad de proteína, cada una con propiedades y características diferentes. Existen investigaciones de fraccionamiento proteico según la solubilidad de Osborne que utilizan diferentes solventes, tiempos de extracción y metodologías para obtener albuminas, globulinas, prolaminas y glutelinas. En el presente estudio se evaluó cinco técnicas de fraccionamiento proteico según solubilidad de Osborne empleadas en la harina de kiwicha, se consideró metodología, solventes y tiempo de extracción óptimos de las técnicas evaluadas para obtener fracciones con mayores contenidos proteínicos y por consiguiente mayores rendimientos porcentuales (Figura 1). De esta manera, se observó en el fraccionamiento proteico de la harina de semilla de kañihua Ramis y Cupi-Sayhua mayores rendimientos porcentuales $(p<0,05)$ con la técnica de Rodríguez y et.a ${ }^{28}$ para albuminas y glutelinas, y Barba de la Rosa y et.al ${ }^{18}$ para globulinas 7S, globulinas 11S y prolaminas, en comparación a las otras técnicas evaluadas. Además, la fracción prolaminas mostró menor rendimiento debido a su bajo contenido proteico, también reportado en kiwicha y describen que esta fracción sería la responsables de la patología celiaca ${ }^{10}$, en consecuencia las fracciones proteicas de kañihua serían una alternativa alimentaria para los intolerantes al gluten. Así mismo, la extracción secuencial durante $1 \mathrm{~h}$ de las fracciones proteicas de la harina de kañihua en ambas variedades, mostró mayor rendimiento porcentual en comparación a 2, 4 y $8 \mathrm{~h}$ (Figura 2), tiempo análogo al reportado en la obtención de fracciones proteicas en la semilla de kiwicha ${ }^{18,20,26,27,40}$ pero diferente al de Rodríguez y et.al ${ }^{28}$, que reportaron mejores resultados a $14 \mathrm{~h}$ de extracción de las fracciones proteicas obtenidas en las hojas de kiwicha.

La obtención de las fracciones proteicas de kañihua en condiciones óptimas de solventes, metodologías y tiempo, mostraron el contenido y distribución proteica para kañihua Ramis en albuminas (15,4\%), globulinas $7 \mathrm{~S}(24,1 \%)$, globulinas $11 \mathrm{~S}(25,7 \%)$, prolaminas $(9,6 \%)$ y glutelinas $(22,9 \%)$; y para kañihua Cupi-Sayhua en albuminas (15,8\%), globulinas $7 \mathrm{~S}(26,3 \%)$, globulinas $11 \mathrm{~S}(26,7 \%)$, prolaminas $(9,9 \%)$ y glutelinas (21,5\%) (Tabla 3). Estos resultados fueron mayores a los reportados en las semillas de kañihua en albúminas y globulinas $(45,0 \%)$, prolaminas $(28,0 \%)$, glutelinas y proteínas insolubles $(31,0 \%)$; con diferencias en las prolaminas y glutelinas lo cual se explicaría por la metodología de extracción utilizada y la inclusión de proteínas insolubles ${ }^{21}$. En quinua, reportaron en las semillas albúminas y globulinas $(45,0 \%)$ prolaminas $(23,0 \%)$, glutelinas y proteínas insolubles $(32,0 \%)^{35,42}$. En kiwicha, describieron en las hojas, albuminas $(73,4 \%)$, globulinas $(65 \%)$, prolaminas $(6,5 \%)$ y glutelinas $(60 \%)^{28}$. En las semillas, reportaron albuminas + NNP como la fracción más abundante con 30 a 50\%, globulinas 5 a 16\%, prolaminas 0,9 a $2 \%$, y glutelinas 16 a $41 \%{ }^{18,27}$. De manera similar, Búcaro y Bressani ${ }^{40}$, describieron albuminas + NNP, globulinas, prolaminas y glutelinas 29,2; 24,5; 0,95 y 28,8\%, respectivamente. En otras investigaciones en semilla de kiwicha que extrajeron sólo albuminas sin considerar NNP, reportaron albuminas $33,0 \%$, globulinas $17,5 \%$, prolaminas $6,9 \%$ y glutelinas $1,7 \%{ }^{20}$. También, informaron el contenido de albuminas $20,0 \%$, globulinas $19,1 \%$, prolaminas $0,9 \%$ y glutelinas $30,9 \%{ }^{43}$. A diferencia, de la investigación que describió albuminas $10,4 \%$, globulinas $0,8 \%$, prolaminas $0,6 \%$ y glutelinas $22,5 \%{ }^{44}$. Los altos contenidos de albuminas y globulinas, seguido de glutelinas son comparables con la soya y en menor proporción con los cereales ${ }^{45}$. 
En el presente estudio, para la extracción de la fracción albuminas se usó la proporción de harina/solvente de 1:10 $(\mathrm{p} / \mathrm{v})$, similar al reportado en semilla de kiwicha ${ }^{18,19,26,27,46-48} ; \mathrm{y}$ diferente al empleado en hojas en la proporción 1:30 (p/v) ${ }^{28}$. En globulinas $7 \mathrm{~S}$ y $11 \mathrm{~S}$, se obtuvo los mayores rendimientos con los solventes $\mathrm{Na}_{2} \mathrm{HPO}_{4}$ y NaCl , igual a los empleados por Barba de la Rosa y et.al ${ }^{18}$, Silva-Sánchez y et.al ${ }^{19}$, ParedesLópez $^{49}$, y Konishi y et.al ${ }^{48}$, en contraste del $\mathrm{K}_{2} \mathrm{HPO}_{4}$ y $\mathrm{NaCl}$ reportado por Maldonado-Cervantes y et.al ${ }^{26}$. Así mismo, describieron que $\mathrm{Na}_{2} \mathrm{HPO}_{4}$ y $\mathrm{NaCl}$ mejoró la solubilidad de globulinas en guisantes ${ }^{50}$. En prolaminas el mejor rendimiento se mostró con 2-propanol al 70\% ${ }^{18,27-28,40}$. En glutelinas con el solvente $\mathrm{NaOH}$ se obtuvo mejores resultados, análogos a los descritos en kiwicha ${ }^{26-28,40,51}$.

En la investigación de Barba de la Rosa y et.al ${ }^{18}$ mencionaron la importancia de la deslipidización de la harina integral para estudios cualitativos de fracciones proteicas, debido a que mejoró significativamente la calidad de los patrones electroforéticos. En el presente estudio el perfil electroforético de las fracciones proteicas de la harina de la semilla de kañihua Cupi-Sayhua (Figura 3), mostró patrones similares en número de bandas y diferentes en concentraciones, siendo más intensos las bandas de Cupi-Sayhua en comparación a la variedad Ramis. Perfil similar, al observado en el $\operatorname{arroz}^{52}$ y kiwicha ${ }^{18-20,27,44,45,49,53}$.

En la fracción albumina en ambas variedades de kañihua, presentaron regiones de polipéptidos entre 5 y $95 \mathrm{kDa}$, similar a lo reportado en kiwicha y describieron una banda típica alrededor de $36 \mathrm{kDa}^{18,27,54}$, otros autores mostraron bandas alrededor de $35 \mathrm{kDa}$ ricas en lisina ${ }^{55}, 18 \mathrm{kDa}$ ricas en metionina ${ }^{51}$, y esta fracción en kiwicha fue comparable en su composición de aminoácidos a la albumina del huevo y fue utilizada como sustitutivo en la industria panificadora ${ }^{19}$. También, se han reportado a partir de esta fracción péptidos bioactivos con actividad anti-hipertensiva, antioxidante y anti-cancerígena ${ }^{20,53}$.

En el presente estudio, la globulina $7 \mathrm{~S}$ presentó regiones entre 4 y $92 \mathrm{kDa}$, y en globulina $11 \mathrm{~S}$ entre 4 y $37 \mathrm{kDa}$, en el grano kiwicha reportaron en globulinas una banda alrededor de $55 \mathrm{kDa}$ denominada precursora de globulinas ${ }^{27}$, y otras regiones entre 22 y $38 \mathrm{kDa}$. Además, se describieron péptidos bioactivos derivados de las globulinas con actividad inhibitoria de la enzima convertidora de angiotensina ${ }^{19,27,53}$. De manera similar, se reportaron bandas de globulinas entre 50 y $90 \mathrm{kDa}$ en el guisante convicilin y soya ${ }^{56,57}$, la migración de las bandas de globulina $7 \mathrm{~S}$ y $11 \mathrm{~S}$ dependen principalmente de la fuerza iónica de sus agentes extractores ${ }^{50}$.

En la fracción de prolaminas en kañihua Ramis y CupiSayhua se observó varias bandas entre 6,5 y 22 kDa, similar a lo reportado en kiwicha ${ }^{45}$, denominadas bandas de bajo peso molecular (10 a $14 \mathrm{kDa}$ ) y solubles en alcohol. A diferencia, otros autores reportaron bandas entre 24 y $67 \mathrm{kDa}$ y de elevado peso molecular $(>94 \mathrm{kDa})^{27}$.

En la fracción de glutelina en ambas variedades de kañihua se presentaron regiones entre 6 y $230 \mathrm{kDa}$, similar a lo reportado para kiwicha por Barba de la Rosa y et.a ${ }^{27} y$ Tovar-Pérez y et.al ${ }^{53}$. Al igual que las prolaminas, presentan regiones de elevada masa molecular unidos por puentes disulfuro que dificultan o no permiten el ingreso de las muestras al gel de poliacrilamida ${ }^{27}$. Así mismo, se describieron péptidos bioactivos de ésta fracción con actividad anti-hipertensiva y anti-cancerígena ${ }^{18,19,26,53,58}$.

Es importante mencionar que las investigaciones de nutracéuticos de kañihua hasta el momento fueron realizados en concentrados proteicos ${ }^{13,15}$, y ninguno en fracciones proteicas, fuentes ricas en contenido proteínico y por consiguiente en péptidos bioactivos.

\section{CONCLUSIONES}

Se observó un mayor contenido proteínico en la harina de la semilla en ambas variedades de kañihua, siendo mayor en Cupi-Sayhua, y en sus fracciones proteicas, en comparación a kiwicha y trigo. A la vez, se encontró mayores rendimientos porcentuales en el fraccionamiento proteico con la técnica de Rodríguez y et.a ${ }^{28}$, para albuminas y glutelinas, y con el de Barba de la Rosa y et.alli, para globulinas y prolaminas. El tiempo óptimo que mostró mayor rendimiento porcentual de extracción secuencial de las fracciones proteicas fue de 1 h. La composición y distribución de proteínas solubles de kañihua Ramis y Cupi-Sayhua con los solventes, metodologías y tiempo óptimo fueron: albuminas $15,4 \pm 0,3$ y $15,8 \pm 0,3 \%$; globulinas $7 \mathrm{~S} 24,1 \pm 0,5$ y $26,3 \pm 1,0 \%$; globulinas $11 \mathrm{~S} 25,7$ $\pm 1,0$ y $26,7 \pm 1,0 \%$; prolaminas $9,6 \pm 0,1$ y $9,9 \pm 0,5 \%$ y glutelinas $22,9 \pm 0,1$ y $21,5 \pm 1,4 \%$, respectivamente. En el perfil electroforético se observó patrones similares en número de bandas y diferentes en concentraciones en las dos variedades de kañihua, encontrándose bandas más intensas en kañihua Cupi-Sayhua.

Agradecimientos. Esta investigación fue financiada por CIENCIACTIVA-CONCYTEC en el marco del contrato 007-2014-FONDECYT. Agradecemos a Mg. Inés Arnao del Centro de Investigación de Bioquímica y Nutrición-UNMSM por la revisión del artículo y Mg. Elizabeth Carranza del Instituto Nacional de Biología Andina, por facilitar su equipamiento.

\section{RESUMEN}

La kañihua (Chenopodium pallidicaule Aellen) es una Chenopodiacea de la región andina, que contiene entre 15 y $19 \%$ de proteínas, con aminoácidos esenciales. El objetivo del presente estudio fue fraccionar y caracterizar electroforéticamente las proteínas de la semilla de kañihua variedades Ramis y Cupi-Sayhua. En harina integral se realizó el análisis proximal y fraccionamiento, luego, la harina se fraccionó mediante cinco técnicas según la solubilidad de Osborne para obtener albuminas, globulinas, prolaminas y glutelinas. Se optimizó la metodología, solventes y tiempo de extracción; e identificaron los perfiles electroforéticos de las fracciones. El mayor contenido proteínico $(p \leq 0,05)$ fue de la harina de kañihua y sus fracciones proteicas, en comparación a kiwicha y trigo. El mayor rendimiento porcentual $(p \leq 0,05)$ durante 1 h de extracción secuencial de las fracciones proteicas, se obtuvo con la técnica 
de Rodríguez y et.al., para albuminas y glutelinas, y con la técnica de Barba de la Rosa y et.al., para globulinas y prolaminas. Se encontró en kañihua Ramis y Cupi-Sayhua,

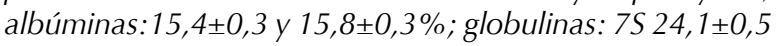
y $26,3 \pm 1,0 \%$; globulinas $115: 25,7 \pm 1,0$ y $26,7 \pm 1,0 \%$; prolaminas: 9, $6 \pm 0,1$ y 9,9 $90,5 \%$ y glutelinas: $22,9 \pm 0,1$ y $21,5 \pm 1,4 \%$, respectivamente. El perfil electroforético mostró patrones similares en número de bandas y diferentes en concentración en ambas variedades.

Palabras clave: Fraccionamiento; electroforesis; proteínas; Chenopodium pallidicaule Aellen.

\section{BIBLIOGRAFIA}

1. Mujica A, Jacobsen S, Ortiz R, Canahua A, Apaza V, Aguilar $P$, Dupeyrat $R$. The Kanihua in human nutrition. Research Institute of the UNA. Puno, Perú: Postgraduate school, UNA, INIA, CARE, PUNO, y CIP. Ed; 2002.

2. Callisaya A, Alvarado A. Isolated protein of high andean grain chenopodiaceas quinua "Chenopodium quinoa" - canahua "Chenopodium pallidicaule" by isoelectric precipitation, Rev Boliviana Quim 2009; 26(1): 12-20.

3. Fritz $M$, Vecchi B, Rinaldi $G$, Añon M. Amarant seed protein hidrolysates have in vitro and in vitro antihypertensive activity, Food Chem 2011; 126(3): 78-84.

4. Tapia M, Fries A. Field guide to andean crops. Lima: FAO \& ANPE ed; 2007.

5. Rastogi A, Shukla S. Amaranth: a new millenium crop of nutraceutical values, Crit Rev Food Sci Nutr 2013; 53(2): 109-125.

6. Rivera R. Andean cultures in Peru. Research and development prospects. Perú: Minerva ed; 1995, p. 417.

7. Mujica A, Chura E. Cultivation of andean grains and cereals. Academic Vice-Rectorade and University Research office. Puno, Perú: UNA ed; 2012, p. 103-132.

8. Repo-Carrasco, R. Andean indigenous food crops. Nutritional value and bioactive compounds [Doctoral thesis]. Turku. Finland: University of Turku, Departament of Biochemistry and Food Chemistry; 2011.

9. FAO/WHO/UNU. Protein and amino acid requirements in human nutrition report of a joint WHO/FAO/UNU, expert consulation technical report series, No 935, WHO, Genera, 2007, p. 50.

10. Mujica A, Moscoso G, Zavaleta A, Canahua A, Chura E, Vignale $D$. Medicinal uses and ancestral nutraceutical knowledge of Andean grains: Quinoa (Chenopodium quinoa Willd) and kanihua (Chenopodium pallidicaule Aellen) in the andean zone. VI International Congress of Etnobotany. In: Summaries The wealth of a legacy. Córdoba, España; 2014. p. 17-21.

11. Rastrelli L, De Simone F, Schettino O, Dini A. Constituents of Chenopodium pallidicaule (Canihua) seeds: isolation and characterization of new triterpene saponins, I Agric Food Chem 1996; 44(1): 3528-3533.

12. Hernández-Ledesma B, Contreras M, Recio I. Antihypertensive peptides: Production, bioavailability and incorporation into foods. Adv, Colloid Interface Sci 2011; 165(1): 23-35.

13. Espinoza E. Antioxidant and antihypertensive activity of canihua concentrated protein, Rev NSB 2017; 43(1): 1-15.

14. Masayo A, Watanabe K. Funtional and bioactive propierties of quinoa and amaranth, Food Sci Technol Res 2010; 16(2): 63-68.

15. Peñarrieta $M$, Alvarado A, Akesson B, Bergenstahl B. Total antioxidant capacity and content of flavonoids and other phenolic compounds in canihua (Chenopodium pallidicaule): An Andean pseudocereal, Mol Nutr Food Res 2008; 52(1): 708-717.

16. Mujica A, Moscoso G, Zavaleta A, Canahua A, Juarez R, Chambi $W$, Vignale $D$. Medicinal uses and ancestral nutraceutical knowledge of andean grains: Quinoa (Chenopodium quinoa Willd), Kanihua (Chenopodium pallidicaule Aellen), Andean tubercules: Izaño (Tropaeolum tuberosum $R$ y $P$ ) Olluco (Ullucus tuberosus Loz.), Oca (Oxalis tuberosa Mol.) and wild relatives in the peruvian altiplano. In: III Latin american medicinal plants- Antonio Brack Egg Congress from august 12 to 14 lquitos, Perú; 2015.

17. Mengoni A, Quiroga A, Añon M. Purification and characterization of a lectin of amaranthus hypochondriacus, an antiproliferative compound, INNOTEC 2016; 11(1): 27-35.

18. Barba de la Rosa A, Fomsgaard I, Laursen B, Mortensen A, Olvera-Martínez J, Silva-Sánchez C, Mendoza-Herrera A, De León-Rodríguez A, González-Castañeda J. Amaranth (Amaranthus hypochondriacus) as an alternative crop for sustainable food production: phenolic acids and flavonoids with potential impact on its nutraceutical quality, I Cereal Sci 2009; 49: 117-121.

19. Silva-Sánchez C, Barba de la Rosa A, León-Galván $M$, de Lumen B, De León-Rodríguez A, and González de Mejía E. Bioactive peptides in amaranth (Amaranthus hypochondriacus) seed, I Agric Food Chem 2008; 56: 1233-1240.

20. Soriano-Santos J, Iwabuchi S, Fujimoto K. Solubility of amaranth seed proteins in sodium sulphate and sodium chloride: the main factor in quantitative extraction for analysis, Int J Food Sci and Technol. 1992;27:337-346.

21. Scarpati Z, Briceño O. Evaluation of the chemical composition (nutritional composition) of some quinoa entries (Chenopodium quinoa Wild) of the germplasm bank of the Technical National University of the Altiplano (Perú), An Cient UNALM 1980; 18: 125-134.

22. Osborne TB. The vegetable proteins. Monographs on Biochemistry. 2a edn. Longmans, Green and Co, London; 1924. p. 154.

23. Sabbione A, Scilingo A, Añón M. Potential antithrombotic activity detected in Amaranth proteins and its hydrolysates, LWT-Food Sci Technol 2015; 60(1): 171-177.

24. AOAC. Official Methods of analysis, 18 th ed. Association of Official Analytical Chemists. Washington, USA, 2005.

25. Kirk R, Sawyer R, Egan H. Composition and analysis of Pearson of foods. 2da ed. Continental publishing company SA de CV. México, 1996.

26. Maldonado-Cervantes $E$, Jin H, León F, Barreda A, De León A, Gonzales de Mejia E, De lumen B, Barba de la Rosa A. Amaranth lunasin-like peptide internalizes into the cell nucleus and inhibits chemical carcinogen-induced transformation of NIH-3T3 cells, Peptides 2010; 31: 1635-1642.

27. Barba de la Rosa A, Gueguen J, Paredes O, Viroben G. Fractionation procedures, electrophoretic characterization and amino acid composition of amaranth seed protein, J Agric Food Chem 1992; 40: 931-936.

28. Rodríguez P, Pérez E, Guzman R, Dofour D. Characterization of the proteins fractions extracted from leaves of Amaranthus dubius (Amaranthus spp), African J Food Sci 2011; 5(7): 417424.

29. Bradford M. A rapid and sensitive method for the quantization of microgram quantities of protein utilizing the principle of protein-dye binding, Anal Biochem 1976; 72: 248-254. 
30. Laemmli UK. Cleavage of structural proteins during the assembly of the head of bacteriophage T4, Nature 1970; 227: 680-685.

31. Gomez A. Obtaining peptides with inhibitory activity of the angiotensin I converting enzyme from protein hydrolysates of the amaranth fraction (Amaranthus hypochondriacus L) [Thesis]. Oaxaca, Mexico: Papaloapan University, Faculty of food; 2014.

32. Giuliani A, Hintermann F, Rojas W, Padulosi S. Biodiversity of Andean grains: balancing market potential and sustainable livelihoods. Rome, Italia: Biodiversity International ed; 2012.

33. Marti A, BockJE, Pagani MA, Ismail B, Seetharaman k. Structural characterization of proteins in wheat flour doughs enriched with intermediate wheatgrass (Thinopyrum intermedium) flour, Food Chem 2016; 194: 994-1002.

34. Gil-Hernández A. Cereals and derived products. In: Nutrition treaty. Vol 2. Nutrition composition and quality of foods. México: Panamericana ed; 2010.

35. Asao M, Watanabe K. Functional and bioactive properties of quinoa and amaranth, Food Sci and Technol Res 2010;1 6(2): 163-168.

36. Repo-Carrasco-Valencia $R$, Encina C, Binaghi $M$, Greco $C$, Ronayre $P$. Effects of roasting and boiling of quinoa, kiwicha and kaniwa on composition and availability of minerals in vitro, I Sci Food Agric 2010; 90: 2068-2073.

37. USDA, Wheat: In National Nutrient Database for Standard Reference Release 28. United States Department of Agricultural Research Service; 2015, August 20. Retrieved from: http:// ndb.nal.usda.gov/ndb/foods

38. Repo-Carrasco R. Andean indigenous food crops: nutritional value and bioactive compounds. Painosalama Oy-Turku Finland: Department of Biochemistry and Food Chemistry, University of Turku; 2011. p.145.

39. Tang Y, Tsao R. Phytochemicals in quinoa and amaranth grains and their antioxidant, anti-inflammatory and potential health beneficial effects:a review, Mol Nutr Food Res 2017; 1600767.

40. Búcaro M, Bressani R. Distribution of the protein in physical fractions of the grinding and sieving of amaranth grain, Arch Latinoam Nutr 2002; 52(2): 167-171.

41. Luna S, Medina S, Cruz A, Paredes-López O. Modification of the amaranth $11 S$ globulin storage protein to produce an angiotensin I converting enzyme inhibitory peptide, and its expression in E. coli, J Biotechnol 2010; 148(4): 240-247.

42. Filho A, Pirozi M, Borges J, Pinheiro S, Chaves J, Coimbra J. Quinoa: Nutrition, functional, and antinutritional aspects, Crit Rev Food Sci Nutr 2017; 57(8): 1618-1630.

43. Bressani $R$, García-Vela L. Protein fractions in amaranth grain and their chemical characterization, I Agric Food Chem 1990;38 1205-1209.

44. Konishi $Y$, Iyota $H$, Yoshida K, Moritani J, Inoue T, Nishimura $N$, Nomura T. Effect of moisture content on the expansion volume of popped Amaranth seeds by hot air and superheated steam using a fluidized bed system, Biosci Biotechnol Biochem 2004; 68(109): 2186-2189.

45. Pa?ko P, Barto? H, Zagrodzki P, Gorinstein S. Effect of amaranth seeds (Amaranthus cruentus) in the diet on some biochemical parameters and essential trace elements in blood of high fructose-fed rats, Nat Prod Res 2011; 25(8): 844-849.

46. Lado M, Burini J, Rinaldi G, Añón M, Tironi V. Effects of the Dietary Addition of Amaranth (Amaranthus mantegazzianus) Protein Isolate on Antioxidant Status, Lipid Profiles and Blood Pressure of Rats, Plant Foods Hum Nutr 2015; 70(1): 371-379.

47. Montoya-Rodríguez A, Milán-Carrillo J, Reyes-Moreno C, González de Mejía E. Characterization of peptides found in unprocessed and extruded amaranth (Amaranthus hypochondriacus) pepsin/pancreatin hydrolysates. Int J Mol Sci. 2015; 16(4): 8536-8554.

48. Konishi Y, Horikawa K, Oku Y, Azumaya J, Nakatani N. Extraction of two albumin fractions from amaranth grains: comparison of some physicochemical properties and the putative localization in the grains. Agric Biol Chem 1991; 55: 1745-1750.

49. Paredes-López O, Guevara F, Bello A. The magical foods of mesoamerican indigenous cultures. Mexico: CONACYT; 2006. p. 197.

50. Guéguen J, Viroben G, Noireaux P, Subirade M. Influence of plasticizers and treatments on the properties of films from pea proteins- Industr Crops Prod 1998; 7: 149-157.

51. Segura-Nieto $M$, Shewry P, Paredes-López O. Globulins of pseudocereals: Amaranth, quinoa, and buckwheat. In: Seed Proteins. Mexico: Shewry P. Casey R; 1999. p.453-475.

52. Pinciroli M. Rice proteins: structural and functional properties [Master Thesis]. Argentina: National University of the Silver, Faculty of Agrarian and Forest Sciences; 2010.

53. Tovar-Pérez E, Guerrero-Legarreta I, Farrés-Gonzáles A, Soriano-Santos J. Angiotensin I-converting enzyme-inhibitory peptide fractions from albumin 1 and globulin as obtained of amaranth grain. J Agric Food Chem 2009; 116: 437-444.

54. Mora-Escobedo R, Paredes-López O, Ordorica-Falomir C. Characterization of albumins and globulins from amaranth. J Lebensm Wiss Technol 1990; 23(6): 484-487.

55. Villanueva O, Arnao I. Purification of a lysine rich $35 \mathrm{kDa}$ protein from the albumin fraction of (kiwicha) Amaranthus caudatus. An Fac Med 2007; 68(4): 344-349.

56. O'Kane F, Happe R, Vereijken J, Gruppen H, Van Boekel M. Characterization of pea vicilin. 1. Denoting convicilin as the alpha-subunit of the Pisum vicilin family. J Agric Food Chem 2004; 52(10): 3141-3148.

57. Singh A, Meena M, Kumar D, Dubey A, Hassan I. Structural and functional analysis of various globulin proteins from soy seed. Crit Rev Food Sci Nutr 2015; 55(11): 1491-1502.

58. Ranilla LG, Apostolidis E, Genovese MI, Lajolo FM, Shetty K. Evaluation of indigenous grains from the Peruvian Andean region for antidiabetes and antihypertension potential using in vitro methods. J Med Food 2009; 12(4): 704-713. 